\title{
A CHARACTERIZATION OF CERTAIN WEAKLY PSEUDOCONVEX DOMAINS
}

\author{
Dedicated to Professor Fuichi Uchida on his sixtieth birthday
}

\author{
AKIo Kodama
}

(Received July 8, 1997, revised March 9, 1998)

\begin{abstract}
By making use of well-known extension theorems on holomorphic mappings and CR-mappings and applying Webster's CR-invariant metrics, we give a characterization of certain weakly pseudoconvex domains from the viewpoint of biholomorphic automorphism groups.
\end{abstract}

Introduction. This is a continuation of our previous paper [11], and we retain the terminology and notation there.

Let $D$ be a bounded domain in $C^{n}$ and let $p \in \partial D$. Then we say that the condition (*) is fulfilled for $(D, p)$ if

there exists a compact set $K$ in $D$, a sequence $\left\{k_{v}\right\}$ in $K$ and a sequence $\left\{\varphi_{v}\right\}$ in $\operatorname{Aut}(D)$ such that $\lim _{v \rightarrow \infty} \varphi_{v}\left(k_{v}\right)=p$.

Now assume that the condition $(*)$ is fulfilled for $(D, p)$. Then we may ask if it is possible to determine the global structure of $D$ from the local shape of the boundary $\partial D$ near $p$. Certainly, it is impossible without any further assumption, as one may see in the examples such as the direct product of the open unit disk in $C$ and an arbitrary bounded domain in $C^{n-1}$. As for this problem, it was shown by Wong [25] that if $D$ is a strictly pseudoconvex domain in $C^{n}$ with smooth boundary and the condition $(*)$ is fulfilled for $(D, p)$ for some $p \in \partial D$, then $D$ is biholomorphically equivalent to the open unit ball $B^{n}$ in $C^{n}$. It was later extended by Rosay [20] to the case where $\partial D$ near $p$ is $C^{2}$-smooth and strictly pseudoconvex. It is natural to see what happens when $p$ is a weakly (not strictly) pseudoconvex boundary point of D. It was Greene and Krantz [8] who first dealt with this problem in the category of weakly pseudoconvex domains in $C^{n}$ with globally $C^{n+1}$-smooth boundaries. As a generalization of their result, we obtained in [11] the following characterization of the weakly pseudoconvex domain

$$
E(k, \alpha)=\left\{\left.z \in C^{n}\left|\sum_{i=1}^{k}\right| z_{i}\right|^{2}+\left(\sum_{j=k+1}^{n}\left|z_{j}\right|^{2}\right)^{\alpha}<1\right\},
$$

where $k \in \boldsymbol{Z}$ with $1 \leqq k \leqq n$ and $0<\alpha \in \boldsymbol{R}$, and it is understood that $E(k, \alpha)=B^{n}$ if $k=n$ :

Partly supported by the Japan-US Cooperative Science Program and the Grant-in-Aid for Scientific Research, the Ministry of Education, Science, Sports and Culture, Japan.

1991 Mathematics Subject Classification. Primary 32F15; Secondary 32H99. 
THEOREM K (Kodama [11]). Let $D$ be a bounded domain in $C^{n}$ satisfying the following conditions:

(1) $p=(1,0, \ldots, 0) \in \partial D \cap \partial E(k, \alpha)$;

(2) there is an open neighborhood $U$ of $p$ in $C^{n}$ such that $D \cap U=E(k, \alpha) \cap U$;

(3) the condition $(*)$ is fulfilled for $(D, p)$.

Then $D$ is biholomorphically equivalent to the domain $E(k, \alpha)$.

It should be remarked that, in general, $E(k, \alpha)$ is not geometrically convex and, moreover, its boundary is not smooth at every point $x$ of the form $x=$ $\left(x_{1}, \ldots, x_{k}, 0, \ldots, 0\right)$. Also, noting the fact that such a boundary point $x$ is an accumulation point of the $\operatorname{Aut}(E(k, \alpha))$-orbit passing through the origin of $C^{n}$, one sees that exactly the same conclusion in Theorem $\mathrm{K}$ remains valid for an arbitrary point $x=\left(x_{1}, \ldots, x_{k}, 0, \ldots, 0\right) \in \partial D \cap \partial E(k, \alpha)$ as well as $p=(1,0, \ldots, 0)$. This theorem was later extended by Kodama, Krantz and Ma [15] to a more general domain, called a generalized complex ellipsoid,

$$
E\left(n ; n_{1}, \ldots, n_{s} ; p_{1}, \ldots, p_{s}\right)=\left\{\left(z_{1}, \ldots, z_{s}\right) \in C^{n_{1}} \times \cdots \times\left. C^{n_{s}}\left|\sum_{i=1}^{s}\right| z_{i}\right|^{2 p_{i}}<1\right\}
$$

in $\boldsymbol{C}^{n}=\boldsymbol{C}^{n_{1}} \times \cdots \times \boldsymbol{C}^{n_{s}}$, where $0<p_{1}, \ldots, p_{s} \in \boldsymbol{R}$ and $0<n_{1}, \ldots, n_{s} \in \boldsymbol{Z}$ with $n=n_{1}+$ $\cdots+n_{s}$, as follows:

Theorem K-K-M (Kodama, Krantz and Ma [15]). Let $D$ be a bounded domain in $C^{n}$ with a point $p \in \partial D$ and $E$ a generalized complex ellipsoid in $C^{n}$ as above. We assume that

(1) $p \in \partial E$ and there is an open neighborhood $U$ of $p$ in $C^{n}$ such that $D \cap U=E \cap U$;

(2) the condition $(*)$ is fulfilled for $(D, p)$ and also for $(E, p)$.

Then $D$ is biholomorphically equivalent to E. In particular, at least one of the exponents $p_{i}$ must be equal to 1 .

In view of Kodama [12], [13] (in which the structure of generalized complex ellipsoids in $C^{n}$ with all $n_{i}=1$ was investigated), it would be natural to ask the following questions: In Theorem K-K-M,

(Q.1) can we remove the condition $(*)$ for $(E, p)$ ?;

(Q.2) can we prove that $D=E$ as sets?

These cannot be answered in full generality at this moment except when all $p_{i}$ 's are positive integers, i.e., the boundary $\partial E$ is real-analytic (cf. [14]). Recall that our proofs there relied heavily upon a result on the localization principle of holomorphic automorphisms of generalized complex ellipsoids $E$ with real analytic boundaries due to Dini and Selvaggi Primicerio [5], [6]. A glance at their proof tells us that the real analyticity of $\partial E$ cannot be avoided with their technique.

The main purpose of this paper is to give partial affirmative answers to the questions (Q.1) and (Q.2) when the boundary $\partial E$ is not necessarily smooth. In fact, we 
consider here exclusively generalized complex ellipsoids $E(n ; k, n-k ; 1, \alpha)=E(k, \alpha)$ with arbitrary real numbers $\alpha>0$ and prove the following theorems, which were announced at the POSTECH International Conference on Several Complex Variables in Pohang, South Korea, 1997:

THEOREM 1. Let $E_{1}=E(k, \alpha), E_{2}=E(l, \beta)$ be generalized complex ellipsoids in $C^{n}$ with arbitrary real numbers $\alpha, \beta>0$ and let $p_{1} \in \partial E_{1}, p_{2} \in \partial E_{2}$. We assume that

(1) $k \leqq n-2$ and $l \leqq n-2$;

(2) there are open neighborhoods $U_{1}$ of $p_{1}, U_{2}$ of $p_{2}$ in $C^{n}$ and a biholomorphic mapping $f: U_{1} \rightarrow U_{2}$ such that $f\left(p_{1}\right)=p_{2}, f\left(U_{1} \cap E_{1}\right)=U_{2} \cap E_{2}$ and $f\left(U_{1} \cap \partial E_{1}\right)=$ $U_{2} \cap \partial E_{2}$.

Then $f$ extends to a biholomorphic mapping $F$ from $E_{1}$ onto $E_{2}$. In particular, we have $(k, \alpha)=(l, \beta)$.

Combining this with a result of Bell [2; Theorem 2], we obtain the following:

COROLlary. Let $E(k, \alpha)$ and $E(l, \beta)$ be generalized complex ellipsoids in $C^{n}$ with $k \leqq n-2, l \leqq n-2$ and assume that $f: E(k, \alpha) \rightarrow E(l, \beta)$ is a proper holomorphic mapping. Then $(k, \alpha)=(l, \beta)$ and $f$ is a biholomorphic automorphism of $E(k, \alpha)$.

THEOREM 2. Let $D$ be a bounded domain in $C^{n}$ and let $E=E(k, \alpha)$ be a generalized complex ellipsoid in $\boldsymbol{C}^{n}$ with $0<\alpha \in \boldsymbol{R}$. We assume that

(1) there exist a point $p \in \partial D \cap \partial E$ and an open neighborhood $U$ of $p$ in $C^{n}$ such that $D \cap U=E \cap U$;

(2) the condition $(*)$ is fulfilled for $(D, p)$.

Then we have $D=E$ as sets.

We would like to remark that the assumption (1) in Theorem 1 is essential. Indeed, consider the generalized complex ellipsoids $E_{1}=\left\{(z, w) \in C \times\left. C|| z\right|^{2}+|w|^{2 \alpha}<1\right\}, E_{2}=B^{2}$ and a branch $f$ of $(z, w) \mapsto\left(z, w^{\alpha}\right)$ defined in a small neighborhood of a point $p_{1}=\left(z_{o}, w_{o}\right) \in \partial E_{1}$ with $w_{o} \neq 0$, where $0<\alpha \in \boldsymbol{R}, \alpha \neq 1$. Then $f$ gives rise to a biholomorphic equivalence between a neighborhood $U_{1}$ of $p_{1}$ and a neighborhood $U_{2}$ of $p_{2}:=f\left(p_{1}\right) \in \partial E_{2}$ satisfying the condition (2) in Theorem 1; however, it is clear that $f$ cannot be continued to a biholomorphic mapping from $E_{1}$ onto $E_{2}$. Also, considering the special case $\alpha=\beta=1$ in the corollary above, we see that every proper holomorphic self-mapping of the unit ball $B^{n}$ must be a biholomorphic automorphism of $B^{n}$. This is just a well-known theorem of Alexander [1].

In Section 1, by making use of Rudin's extension theorem [21; p. 311] on holomorphic mappings defined near boundary points of $B^{n}$, we show some properties of generalized complex ellipsoids $E(k, \alpha)$, which will be a key step to the proofs of our theorems. After this preparation, Theorems 1 and 2 will be proved in Sections 2 and 3 , respectively. Our proofs here are based on some extension theorems on proper holomorphic mappings and CR-mappings obtained by Forstnerič and Rosay [7], Pinchuk [18], [19], Bell [3], and also on the existence of Webster's CR-invariant metrics 
on strictly pseudoconvex real analytic hypersurfaces in $C^{n}$ without umbilical points [22], [23].

The author would like to express his thanks to Professors Junjiro Noguchi and Kang-Tae Kim for their useful comments on the subject of this paper.

1. A key lemma. For later purpose, we prove some facts on the structure of the model spaces $E(k, \alpha)$ with arbitrary real numbers $\alpha>0$.

Throughout the rest of this paper, we use the following notation: For a point $z=\left(z_{1}, \ldots, z_{n}\right) \in C^{n}$ and for a domain $E(k, \alpha)$, we set $z^{\prime}=\left(z_{1}, \ldots, z_{k}\right), z^{\prime \prime}=\left(z_{k+1}, \ldots, z_{n}\right)$, $E=E(k, \alpha)$ and

$$
\partial^{*} E=\left\{\left(z^{\prime}, z^{\prime \prime}\right) \in C^{k} \times\left. C^{n-k}\left|z^{\prime \prime} \neq 0,\right| z^{\prime}\right|^{2}+\left|z^{\prime \prime}\right|^{2 \alpha}=1\right\}
$$

which is an open dense subset of $\partial E$. Then, by using the facts in the previous paper [11; Section 1], the following assertions are easily proved:

(1.1) $\partial^{*} E$ is a connected, strictly pseudoconvex, real analytic hypersurface in $\boldsymbol{C}^{n}$; moreover, it is simply connected if $k \leqq n-2$ [9; p. 346].

(1.2) $\operatorname{Aut}(E)$ can be regarded as a subgroup of $\operatorname{Aut}\left(B^{k} \times C^{n-k}\right)$.

(1.3) $\operatorname{Aut}(E) \cdot \partial^{*} E=\partial^{*} E$ and $\operatorname{Aut}(E)$ acts transitively on $\partial^{*} E$ as a real analytic CR-automorphism group of $\partial^{*} E$.

The following lemma will play a crucial role in our proofs of Theorems 1 and 2 .

LEMma. Let $E=E(k, \alpha)$ be a generalized complex ellipsoid in $C^{n}$ with $k \leqq n-2$ and let $p \in \partial^{*} E$. Assume that there are an open neighborhood $U$ of $p$ in $C^{n}$ and a biholomorphic mapping $f$ from $U$ into $C^{n}$ such that

$$
U \cap \partial E=U \cap \partial^{*} E, f\left(U \cap \partial^{*} E\right)=f(U) \cap \partial B^{n} \text { and } f(U \cap E)=f(U) \cap B^{n} .
$$

Then $f$ extends to a biholomorphic mapping $F: E \rightarrow B^{n}$. In particular, we have $\alpha=1$.

Proof. Since $\partial^{*} E$ is a connected, strictly pseudoconvex, real analytic hypersurface in $C^{n}$ by (1.1), it follows from a result of Pinchuk [18], [19; p. 193] that $f$ can be continued along any path lying in $\partial^{*} E$ as a locally biholomorphic mapping. Since $\partial^{*} E$ is now simply connected by our assumption $k \leqq n-2$, the monodromy theorem guarantees that $f$ extends to a locally biholomorphic mapping $F$ defined on some connected open neighborhood $V$ of $\partial^{*} E$ in $C^{n}$ such that $F\left(\partial^{*} E\right) \subset \partial B^{n}$ and $F(V \cap E) \subset B^{n}$. Now we will proceed in several steps.

(1) F extends to a holomorphic mapping $\tilde{F}$ from $E$ into $B^{n}$. To prove this, take an arbitrary $r$ with $0<r<1$ and put

$$
K_{r}=\left\{\left(z^{\prime}, z^{\prime \prime}\right) \in \boldsymbol{C}^{k} \times\left.\boldsymbol{C}^{n-k}|| z^{\prime}|\leqq r,| z^{\prime}\right|^{2}+\left|z^{\prime \prime}\right|^{2 \alpha}=1\right\} .
$$

Since $K_{r} \subset \partial^{*} E \subset V$ and $K_{r}$ is compact in $V$, one can choose a small $\varepsilon=\varepsilon(r)>0$ in such a way that

$$
U_{r, \varepsilon}:=\left\{\left(z^{\prime}, z^{\prime \prime}\right) \in C^{k} \times\left. C^{n-k}|| z^{\prime}|<r, 1-\varepsilon<| z^{\prime}\right|^{2}+\left|z^{\prime \prime}\right|^{2 \alpha}<1+\varepsilon\right\} \subset V .
$$


Clearly, $U_{r, \varepsilon}$ is a bounded Reinhardt domain in $C^{n}$. Moreover, since $k \leqq n-2$, we have $U_{r, \varepsilon} \cap\left\{z \in C^{n} \mid z_{j}=0\right\} \neq \varnothing$ for $j=1, \ldots, n$. Hence, by a well-known fact [16; p. 15] every component function $F_{j}$ of $F$ has a holomorphic extension $F_{j}^{r}$ to the domain

$$
\hat{U}_{r, \varepsilon}=\left\{\left(z^{\prime}, z^{\prime \prime}\right) \in C^{k} \times\left. C^{n-k}|| z^{\prime}|<r,| z^{\prime}\right|^{2}+\left|z^{\prime \prime}\right|^{2 \alpha}<1+\varepsilon\right\},
$$

the smallest complete Reinhardt domain in $C^{n}$ containing $U_{r, \varepsilon}$. In particular, putting

$$
E_{r}=\left\{\left(z^{\prime}, z^{\prime \prime}\right) \in C^{k} \times\left. C^{n-k}|| z^{\prime}|<r,| z^{\prime}\right|^{2}+\left|z^{\prime \prime}\right|^{2 \alpha}<1\right\},
$$

we see that $F=\left(F_{1}, \ldots, F_{n}\right)$ has a holomorphic extension $F^{r}:=\left(F_{1}^{r}, \ldots, F_{n}^{r}\right)$ to $E_{r} \cup V$. Note that $E_{r} \subset E_{s}$ for $0<r<s<1, \bigcup_{0<r<1} E_{r}=E$ and that the holomorphic extensions $F^{r}$ are uniquely determined by the values of $F$ on a small neighborhood of the point $(0, \ldots, 0,1) \in V \cap \partial^{*} E$. Then, by standard argument, one can define a holomorphic extension $\widetilde{F}: E \cup V \rightarrow C^{n}$ of $F: V \rightarrow C^{n}$.

Now we wish to show that $\widetilde{F}(E) \subset B^{n}$. For this let us fix an arbitrary point $z_{o}=\left(z_{o}^{\prime}, z_{o}^{\prime \prime}\right) \in E$ and set

$$
E\left(z_{o}\right)=\left\{\left(z_{o}^{\prime}, z^{\prime \prime}\right) \in C^{k} \times\left. C^{n-k}|| z_{o}^{\prime}\right|^{2}+\left|z^{\prime \prime}\right|^{2 \alpha}<1\right\},
$$

which can be regarded as an open ball in $C^{n-k}$. Consider the non-constant, continuous plurisubharmonic function $\psi: z^{\prime \prime} \mapsto-1+\left|\widetilde{F}\left(z_{o}^{\prime}, z^{\prime \prime}\right)\right|^{2}$ defined on some open neighborhood of the closure $\overline{E\left(z_{o}\right)}$ of $E\left(z_{o}\right)$ in $C^{n-k}$. Then $\psi\left(\partial E\left(z_{o}\right)\right)=0$ and $\psi\left(z^{\prime \prime}\right)<0$ on $E\left(z_{o}\right) \cap V$. This, combined with the maximum principle for plurisubharmonic functions, guarantees that $\psi\left(z_{o}^{\prime \prime}\right)<0$, i.e., $\tilde{F}\left(z_{o}\right) \in B^{n}$ and accordingly $\tilde{F}(E) \subset B^{n}$.

(2) There exists a locally injective, real analytic homomorphism $\Phi: \operatorname{Aut}(E) \rightarrow$ $\operatorname{Aut}\left(B^{n}\right)$ such that $\Phi(\sigma) \circ \tilde{F}=\tilde{F} \circ \sigma$ on $E$ for all $\sigma \in \operatorname{Aut}(E)$. Indeed, take an arbitrary $\sigma \in$ $\operatorname{Aut}(E)$. By virtue of (1.2) and (1.3), one can choose an open neighborhood $W$ of the point $p \in \partial^{*} E$ so small that $W \cup \sigma(W) \subset V$ and $\tilde{F}$ is injective on $W$ and on $\sigma(W)$. Let us consider the biholomorphic mapping $\Psi:=\tilde{F} \circ \sigma \circ(\tilde{F} \mid W)^{-1}: \tilde{F}(W) \rightarrow \tilde{F}(\sigma(W))$. By an extension theorem due to Rudin $\left[21 ;\right.$ p. 311] we obtain an element $\tilde{\Psi} \in \operatorname{Aut}\left(B^{n}\right)$ such that $\tilde{\Psi}(z)=\Psi(z)$ for all $z \in \tilde{F}(W \cap E)$. Note that $W \cap E$ and $\tilde{F}(W \cap E)$ are non-empty open subsets of $E$ and $B^{n}$, respectively. Then, by the principle of analytic continuation, we have that $\tilde{\Psi} \circ \tilde{F}=\tilde{F}_{\circ} \sigma$ on $E$ and $\tilde{\Psi}$ is uniquely determined by $\sigma$. Accordingly, one can define a mapping

$$
\Phi: \operatorname{Aut}(E) \rightarrow \operatorname{Aut}\left(B^{n}\right)
$$

by setting $\Phi(\sigma)=\widetilde{\Psi}$ so that $\Phi(\sigma) \circ \widetilde{F}=\tilde{F}_{\circ} \sigma$ on $E$ for all $\sigma \in \operatorname{Aut}(E)$.

It is easy to check that $\Phi$ is a group homomorphism. Once it is shown that $\Phi$ is continuous at the identity element $\operatorname{id}_{E}$ of $\operatorname{Aut}(E)$, it follows that $\Phi$ is real analytic on $\operatorname{Aut}(E)$ (cf. [9; p. 117]). Since the topology of $\operatorname{Aut}(E)$ satisfies the second axiom of countability, we have only to show that $\Phi$ is sequentially continuous at $\mathrm{id}_{E}$. For this let us take an arbitrary sequence $\left\{\sigma_{v}\right\}$ in $\operatorname{Aut}(E)$ which converges to $\mathrm{id}_{E}$ and assume that $\left\{\Phi\left(\sigma_{v}\right)\right\}$ does not converge to the identity element $\operatorname{id}_{B^{n}}$ of $\operatorname{Aut}\left(B^{n}\right)$. Passing to a 
subsequence, we may assume that there is a neighborhood $O$ of $\operatorname{id}_{B^{n}}$ in $\operatorname{Aut}\left(B^{n}\right)$ such that $\Phi\left(\sigma_{v}\right) \notin O$ for all $v$. Pick an arbitrary point $x \in E$. Then $\lim _{v \rightarrow \infty} \Phi\left(\sigma_{v}\right)(\widetilde{F}(x))=$ $\lim _{v \rightarrow \infty} \tilde{F}\left(\sigma_{v}(x)\right)=\tilde{F}(x) \in B^{n}$, which implies that $\left\{\Phi\left(\sigma_{v}\right)(\tilde{F}(x))\right\}$ lies in a compact subset of $B^{n}$. Hence, after taking a subsequence if necessary, we may assume that $\left\{\Phi\left(\sigma_{v}\right)\right\}$ converges to some element $g \in \operatorname{Aut}\left(B^{n}\right)$ (cf. [16; p. 82]). Since $g \notin O$, we see that $g \neq \mathrm{id}_{B^{n}}$. On the other hand, we have $g(\tilde{F}(z))=\lim _{v \rightarrow \infty} \Phi\left(\sigma_{v}\right)(\tilde{F}(z))=\lim _{v \rightarrow \infty} \tilde{F}\left(\sigma_{v}(z)\right)=\tilde{F}(z)$ for all $z \in W \cap E$; consequently, $g=\mathrm{id}_{B^{n}}$ by analytic continuation. This ia a contradiction. Therefore, $\Phi$ is continuous at $\mathrm{id}_{E}$, as desired.

Finally we claim that $\Phi$ is locally injective. It suffices to prove that $\Phi$ is injective in some neighborhood $O$ of $\operatorname{id}_{E}$. To this end, let us select a small open neighborhood $W$ of the point $p \in \partial^{*} E$ in $C^{n}$ and non-empty open subsets $W_{1}, W_{2}$ of $W \cap E$ with the properties: $\tilde{F}$ is injective on $W$, and $W_{1}$ is a relatively compact subset of $W_{2}$. We claim that $O=\left\{\sigma \in \operatorname{Aut}(E) \mid \sigma\left(\bar{W}_{1}\right) \subset W_{2}\right\}$ is what is required. Indeed, it is clear that $O$ is an open neighborhood of $\mathrm{id}_{E}$ in $\operatorname{Aut}(E)$. Moreover, assume that $\Phi\left(\sigma_{1}\right)=\Phi\left(\sigma_{2}\right)$ for $\sigma_{1}, \sigma_{2} \in O$. It follows that $\tilde{F}\left(\sigma_{1}(z)\right)=\Phi\left(\sigma_{1}\right)(\tilde{F}(z))=\Phi\left(\sigma_{2}\right)(\tilde{F}(z))=\tilde{F}\left(\sigma_{2}(z)\right)$ for all $z \in E$. Since $\tilde{F}$ is injective on $W_{2} \subset W$ and since $\sigma_{1}(z), \sigma_{2}(z) \in W_{2}$ for all $z \in W_{1}$, this says that $\sigma_{1}=\sigma_{2}$ on $W_{1}$; and hence $\sigma_{1}=\sigma_{2}$ on $E$ by analytic continuation. Therefore, we have shown that $\Phi$ is locally injective on $\operatorname{Aut}(E)$.

(3) $\tilde{F}: E \rightarrow B^{n}$ is locally injective. Set $S=\{z \in E \mid(J \tilde{F})(z)=0\}$, where $(J \tilde{F})(z)$ denotes the holomorphic Jacobian of $\widetilde{F}$ at $z$. Assume that $S \neq \varnothing$. Then $S$ is a complex analytic subset of $E$ of dimension $n-1$. Once $S \subset\left\{\left(z^{\prime}, z^{\prime \prime}\right) \in C^{k} \times C^{n-k} \mid z^{\prime \prime}=0\right\} \equiv C^{k}$ is shown, we arrive at a contradiction, since $\operatorname{dim} S=n-1>k=\operatorname{dim} C^{k}$ by our assumption. Thus we have only to show that $S \subset C^{k} \times\{0\}$. To this end, take an arbitrary point $x=\left(x^{\prime}, x^{\prime \prime}\right) \in S$ and assume that $x^{\prime \prime} \neq 0$. We may assume that $x$ is a regular point of $S$. Recall that $\widetilde{F} \circ \sigma=\Phi(\sigma) \circ \tilde{F}$ on $E$ for all $\sigma \in \operatorname{Aut}(E)$ by (2). Then

$$
(J \tilde{F})(\sigma(x)) \cdot(J \sigma)(x)=(J \Phi(\sigma))(\tilde{F}(x)) \cdot(J \tilde{F})(x)=0 \quad \text { and } \quad(J \sigma)(x) \neq 0
$$

for all $\sigma \in \operatorname{Aut}(E)$. This means that $\operatorname{Aut}(E) \cdot x$, the $\operatorname{Aut}(E)$-orbit passing through the point $x$, is contained in $S$. This is impossible. Indeed, since $x^{\prime \prime} \neq 0$, one can show by using the explicit expression of $\operatorname{Aut}(E(k, \alpha))$ as in [11; Section 1] that the orbit $\operatorname{Aut}(E) \cdot x$ is a real analytic submanifold of $E$ of real dimension $2 n-1$; on the other hand, $S$ near $x$ is a real analytic submanifold of $E$ of real dimension $2 n-2$. Therefore we conclude that $S \subset C^{k} \times\{0\}$, completing the proof of (3).

Before proceeding further, we need some preparation. First, notice that $B^{n}$ is homogeneous and each element $g \in \operatorname{Aut}\left(B^{n}\right)$ extends to a biholomorphic mapping defined in an open neighborhood of $\bar{B}^{n}$. Thus, shrinking the neighborhood $V$ of $\partial^{*} E$ and replacing $\widetilde{F}$ by a suitable mapping of the form $g \circ \widetilde{F}$ with some $g \in \operatorname{Aut}\left(B^{n}\right)$, if necessary, we may assume that the holomorphic mapping $\tilde{F}: E \cup V \rightarrow C^{n}$ satisfies an additional condition $\tilde{F}(o)=o$, where $o$ stands for the orgin of $C^{n}$. Next, let us consider the toral subgroups $T_{E}$ and $T_{B^{n}}$ of $\operatorname{Aut}(E)$ and $\operatorname{Aut}\left(B^{n}\right)$, respectively, induced by the rotations on $C^{n}$ as follows: 


$$
\left(z_{1}, \ldots, z_{n}\right) \mapsto\left(\left(\exp \sqrt{-1} \theta_{1}\right) z_{1}, \ldots,\left(\exp \sqrt{-1} \theta_{n}\right) z_{n}\right), \quad\left(\theta_{1}, \ldots, \theta_{n}\right) \in \boldsymbol{R}^{n} .
$$

Then $\Phi\left(T_{E}\right)(o)=\Phi\left(T_{E}\right)(\tilde{F}(o))=\tilde{F}\left(T_{E}(o)\right)=\tilde{F}(o)=o$, which says that $\Phi\left(T_{E}\right)$ is contained in the unitary group $U(n)$ of degree $n$ (the isotropy subgroup of $\operatorname{Aut}\left(B^{n}\right)$ at the origin $o$ ). Since $\Phi\left(T_{E}\right)$ as well as $T_{B^{n}}$ is now a maximal torus in $U(n)$ by (2), it is well-known that they are conjugate to each other in $U(n)$, that is, there exists an element $\tau \in U(n)$ such that $\tau \cdot \Phi\left(T_{E}\right) \cdot \tau^{-1}=T_{B^{n}}$. Thus, considering $\tau \circ \tilde{F}, \tau \circ \Phi \circ \tau^{-1}$ instead of $\tilde{F}, \Phi$ if necessary, we may further assume that $\Phi\left(T_{E}\right)=T_{B^{n}}$. Under these assumptions, we claim the following:

(4) $\tilde{F}: E \rightarrow B^{n}$ is, in fact, a biholomorphic mapping. Thanks to the fact (3) one can choose a small open ball $B_{\rho}=\left\{z \in C^{n}|| z \mid<\rho\right\} \subset E$ on which $\tilde{F}$ is injective. Then, since $\tilde{F}\left(B_{\rho}\right)=\tilde{F}\left(T_{E}\left(B_{\rho}\right)\right)=\Phi\left(T_{E}\right)\left(\tilde{F}\left(B_{\rho}\right)\right)=T_{B^{n}}\left(\tilde{F}\left(B_{\rho}\right)\right)$, we see that $\tilde{F}\left(B_{\rho}\right)$ is a bounded Reinhardt domain in $C^{n}$ with center at $\widetilde{F}(o)=o$. Therefore, by a well-known theorem of H. Cartan $\left[21 ;\right.$ p. 24], the restriction $\widetilde{F} \mid B_{\rho}: B_{\rho} \rightarrow \widetilde{F}\left(B_{\rho}\right)$ is a linear transformation. So we may assume that $\tilde{F} \in \operatorname{Aut}\left(C^{n}\right)$. This, combined with the facts that $\tilde{F}\left(\partial^{*} E\right) \subset \partial B^{n}$ and $\partial^{*} E$ is dense in $\partial E$, guarantees that $\tilde{F}(E)=B^{n}$; and hence $\tilde{F}: E \rightarrow B^{n}$ is a biholomorphic mapping. Finally, the assertion $\alpha=1$ follows from a result of Naruki [17]. This completes the proof of the Lemma.

2. Proof of Theorem 1. The proof is divided into three cases as follows:

Case 1. $\alpha=\beta=1$. We have $E_{1}=B^{n}=E_{2}$ in this case; hence our theorem follows at once from Rudin's result [21; p. 311].

Case 2. $\alpha \neq 1, \beta=1$ or $\alpha=1, \beta \neq 1$. We claim that this case does not occur. Indeed, assume the contrary. Since $\partial^{*} E_{1}$ and $\partial^{*} E_{2}$ are open dense subsets of $\partial E_{1}$ and $\partial E_{2}$, respectively, and since $f: U_{1} \rightarrow U_{2}$ is a biholomorphic mapping, we may assume that

$$
p_{1} \in \partial^{*} E_{1}, \quad U_{1} \cap \partial E_{1}=U_{1} \cap \partial^{*} E_{1}, \quad \alpha \neq 1 \quad \text { and } \beta=1 .
$$

In particular, we have $E_{2}=B^{n}$. As an immediate consequence of the Lemma in Section 1 , we now have $\alpha=1$, a contradiction.

Case 3. $\alpha \neq 1, \beta \neq 1$. Without loss of generality, we may assume that $p_{i} \in \partial^{*} E_{i}$ and $U_{i} \cap \partial E_{i}=U_{i} \cap \partial^{*} E_{i}$ for each $i=1,2$. Here, we claim that any strictly pseudoconvex real analytic hypersurface $\partial^{*} E_{i}$ has no umbilical points in the sense of CR-geometry; hence, Webster's CR-invariant Riemannian metric $g_{i}$ can be defined on the whole space $\partial^{*} E_{i}$. (For the notion of umbilical points and Webster's CR-invariant metrics in CR-geometry, see [4]; and also, [22], [23], [24].) To prove our claim, assume that there exists an unbilical point on $\partial^{*} E_{i}$. Then, all the points of $\partial^{*} E_{i}$ are umbilical, since $\operatorname{Aut}\left(E_{i}\right)$ acts transitively on $\partial^{*} E_{i}$ by (1.3). Hence, $\partial^{*} E_{i}$ must be locally biholomorphically equivalent to the sphere $\partial B^{n}$ (see, for example, [22; p. 213]). By the Lemma in Section 1 we conclude that $\alpha=1$ or $\beta=1$ according as $i=1$ or $i=2$. This is a contradiction, as desired. Moreover, we see that $\left(\partial^{*} E_{i}, g_{i}\right)$ is complete as a Riemannian manifold, because $\partial^{*} E_{i}$ is homogeneous under the CR-automorphism group $\operatorname{Aut}\left(E_{i}\right)$. As a result, each $\left(\partial^{*} E_{i}, g_{i}\right)$ 
is a connected and simply connected, complete real analytic Riemannian manifold. On the other hand, $f: U_{1} \cap \partial^{*} E_{1} \rightarrow U_{2} \cap \partial^{*} E_{2}$ is an isometry with respect to the CR-invariant metrics $g_{1}$ and $g_{2}$. By a well-known fact in Riemannian geometry [10; p. 256], $f$ can now be uniquely extended to a global isometry $F:\left(\partial^{*} E_{1}, g_{1}\right) \rightarrow\left(\partial^{*} E_{2}, g_{2}\right)$. It is easily seen that $F: \partial^{*} E_{1} \rightarrow \partial^{*} E_{2}$ is a real analytic CR-diffeomorphism. Accordingly, by a result of Pinchuk [18], [19; p. 186] there are open neighborhoods $V_{1}$ of $\partial^{*} E_{1}$ and $V_{2}$ of $\partial^{*} E_{2}$ in $C^{n}$ such that $F: \partial^{*} E_{1} \rightarrow \partial^{*} E_{2}$ and its inverse $G:=F^{-1}: \partial^{*} E_{2} \rightarrow \partial^{*} E_{1}$ extend to locally biholomorphic mappings written in the same notation $F: V_{1} \rightarrow C^{n}$ and $G: V_{2} \rightarrow C^{n}$ satisfying $F\left(V_{1} \cap E_{1}\right) \subset E_{2}$ and $G\left(V_{2} \cap E_{2}\right) \subset E_{1}$. Hence, in exactly the same way as in (1) of the proof of the Lemma in Section 1, it can be shown that $F$ and $G$ extend to holomorphic mappings $\tilde{F}: E_{1} \rightarrow C^{n}$ and $\tilde{G}: E_{2} \rightarrow C^{n}$. Moreover, replacing $\psi\left(z^{\prime \prime}\right)$ by $\psi_{1}\left(z^{\prime \prime}\right)=\rho_{2}\left(\tilde{F}\left(z_{o}^{\prime}, z^{\prime \prime}\right)\right)$ in (1) of the proof of the Lemma in Section 1, we can prove that $\tilde{F}\left(E_{1}\right) \subset E_{2}$, where $\rho_{2}$ is the continuous plurisubharmonic function on $C^{n}$ defined by $\rho_{2}(z)=-1+\sum_{i=1}^{l}\left|z_{i}\right|^{2}+\left(\sum_{j=l+1}^{n}\left|z_{j}\right|^{2}\right)^{\beta}, z \in C^{n}$. Analogously, we see that $\tilde{G}\left(E_{2}\right) \subset E_{1}$. Since $\tilde{G} \circ \tilde{F}=\mathrm{id}_{E_{1}}$ near $\partial^{*} E_{1}$ and $\tilde{F} \circ \widetilde{G}=\mathrm{id}_{E_{2}}$ near $\partial^{*} E_{2}$, we conclude by analytic continuation that $\tilde{G} \circ \tilde{F}=\mathrm{id}_{E_{1}}$ and $\tilde{F} \circ \widetilde{G}=\mathrm{id}_{E_{2}}$; consequently, $\tilde{F}: E_{1} \rightarrow E_{2}$ is a biholomorphic mapping. Finally the assertion $(k, \alpha)=(l, \beta)$ follows now from Naruki [17], completing the proof of Theorem 1 .

3. Proof of Theorem 2. The case $k=n-1$ is contained in our previous paper [13].

Thus it suffices to prove Theorem 2 when $k \leqq n-2$. We have two cases to consider:

Case 1. The point $p \in \partial D$ is a strictly pseudoconvex boundary point. Hence $D$ is biholomorphically equivalent to $B^{n}$ by a result of Rosay [20]. Fix a biholomorphic mapping $F: D \rightarrow B^{n}$. Using a theorem on the boundary continuity of proper holomorphic mappings due to Forstnerič and Rosay [7], one sees that $F$ extends to a homeomorphism from a connected open neighborhood $M$ of $p$ in $\partial D \cap \partial E$ onto an open subset $M^{\prime}$ of $\partial B^{n}$. Accordingly, by results of Bell [3; Theorem 2], Pinchuk [19; p. 186], the CR-homeomorphism $F: M \rightarrow M^{\prime}$ can be extended to a biholomorphism between some open neighborhoods $O$ of $M$ and $O^{\prime}$ of $M^{\prime}$ in $C^{n}$. Hence, $E=B^{n}$ by the Lemma in Section 1 and $F$ extends to a biholomorphic automorphism $\Phi$ of $B^{n}$ by [21; p. 311]. Set $\Psi=\Phi^{-1} \in \operatorname{Aut}\left(B^{n}\right)$. Then, since $\Psi=F^{-1}$ near $M^{\prime}$, we have that $\Psi=F^{-1}$ on $B^{n}$ by analytic continuation. Thus we obtain that $D=F^{-1}\left(B^{n}\right)=\Psi\left(B^{n}\right)=B^{n}=E$, as desired.

Case 2. The point $p \in \partial D$ is not a strictly pseudoconvex boundary point. The point $p$ must be of the form $p=\left(p_{1}, \ldots, p_{k}, 0, \ldots, 0\right)$ by (1.1). Therefore, it follows at once by Theorem $\mathrm{K}$ in the introduction that there exists a biholomorphic mapping $F: D \rightarrow E$. In exactly the same way as in the proof of [13; Lemma 3], it can be shown that $F$ extends to a homeomorphism from an open subset of $U \cap \partial^{*} E \cap \partial D$ onto an open subset of $\partial^{*} E$. By the same reasoning as above, one can now find points $p_{1} \in U \cap \partial^{*} E, p_{2} \in \partial^{*} E$, open neighborhoods $U_{1}$ of $p_{1}, U_{2}$ of $p_{2}$ in $C^{n}$ and a biholomorphic extension $\tilde{F}: U_{1} \rightarrow U_{2}$ of $F$ satisfying all the conditions in (2) of Theorem 1 . Thus $\widetilde{F}$ extends to a biholomorphic automorphism $\tilde{\Phi}$ of $E$; hence, repeating exactly the same arguments as in Case 1, we 
can show that $D=E$ as sets. This completes the proof of Theorem 2 .

\section{REFERENCES}

[1] H. AleXander, Proper holomorphic mappings in $C^{n}$, Indiana Univ. Math. J. 26 (1977), 137-146.

[2] S. R. BeLl, The Bergman kernel function and proper holomorphic mappings, Trans. Amer. Math. Soc. 270 (1982), 685-691.

[3] S. R. Bell, Local regularity of CR homeomorphisms, Duke Math. J. 57 (1988), 295-300.

[4] D. Burns and S. Shnider, Real hypersurfaces in complex manifolds, Proc. Sympos. Pure Math. 30 (1977), 141-168.

[5] G. Dini and A. Selvaggi Primicerio, Localization principle of automorphisms on generalized pseudoellipsoids, to appear in J. Geom. Anal.

[6] G. Dini and A. Selvaggi Primicerio, Localization principle for a class of Reinhardt domains, Seminari di Geometria 1994-1995, Bologna (1996), 117-127.

[7] F. FORSTNERIČ AND J. P. RoSAY, Localization of the Kobayashi metric and the boundary continuity of proper holomorphic mappings, Math. Ann. 279 (1987), 239-252.

[8] R. E. Greene and S. G. Krantz, Characterizations of certain weakly pseudoconvex domains with non-compact automorphism groups, Lecture Notes in Math. 1268, Springer-Verlag, Berlin, Heidelberg, New York, London, Paris and Tokyo, 1987, 121-157.

[9] S. Helgason, Differential Geometry, Lie Groups, and Symmetric Spaces, Academic Press, New York, London, Toronto, Sydney and San Francisco, 1978.

[10] S. Kobayashi and K. Nomizu, Foundations of Differential Geometry, Volume I, Interscience Publishers, New York and London, 1963.

[11] A. Kodama, Characterizations of certain weakly pseudoconvex domains $E(k, \alpha)$ in $C^{n}$, Tôhoku Math. J. 40 (1988), 343-365.

[12] A. KodAma, A characterization of certain domains with good boundary points in the sense of Greene-Krantz, Kodai Math. J. 12 (1989), 257-269.

[13] A. Kodama, A characterization of certain domains with good boundary points in the sense of Greene-Krantz, II, Tôhoku Math. J. 43 (1991), 9-25.

[14] A. Kodama, A characterization of certain domains with good boundary points in the sense of Greene-Krantz, III, Osaka J. Math. 32 (1995), 1055-1063.

[15] A. Kodama, S. G. Krantz and D. Ma, A characterization of generalized complex ellipsoids in $\boldsymbol{C}^{n}$ and related results, Indiana Univ. Math. J. 41 (1992), 173-195.

[16] R. Narasimhan, Several complex variables, Univ. Chicago Press, Chicago and London, 1971.

[17] I. NARUKI, The holomorphic equivalence problem for a class of Reinhardt domains, Publ. Res. Inst. Math. Sci., Kyoto Univ. 4 (1968), 527-543.

[18] S. I. PINCHuk, On the analytic continuation of holomorphic mappings, Math. USSR Sb. 27 (1975), 375-392.

[19] S. I. PINCHUK, Holomorphic maps in $C^{n}$ and the problem of holomorphic equivalence, Encyclopaedia of Math. Sciences, Vol. 9, G. M. Khenkin, ed., Several Complex Variables III, Springer-Verlag, Berlin, Heidelberg, New York, London, Paris and Tokyo, 1989, 173-200.

[20] J. P. Rosay, Sur une caractérisation de la boule parmi les domaines de $\boldsymbol{C}^{n}$ par son groupe d'automorphismes, Ann. Inst. Fourier (Grenoble) 29 (1979), 91-97.

[21] W. Rudin, Function Theory in the Unit Ball of $C^{n}$, Springer-Verlag, New York, Heidelberg and Berlin, 1980.

[22] A. E. Tumanov, The geometry of CR-manifolds, Encyclopaedia of Math. Sciences, Vol. 9, G. M. Khenkin, ed., Several Complex Variables III, Springer-Verlag, Berlin, Heidelberg, New York, 
London, Paris and Tokyo, 1989, 201-222.

[23] S. M. Webster, Pseudo-hermitian structures on a real hypersurface, J. Diff. Geom. 13 (1978), 25-41.

[24] S. M. Webster, On the transformation group of a real hypersurface, Trans. Amer. Math. Soc. 231 (1977), 179-190.

[25] B. Wong, Characterization of the unit ball in $C^{n}$ by its automorphism group, Invent. Math. 41 (1977), 253-257.

Department of Mathematics

FACUlTy OF SCIENCE

KanaZaWa University

KANAZAWA 920-1192

JAPAN 